\title{
Correction to: Towards Comprehensive Security Analysis of Hidden Services Using Binding Guard Relays
}

\author{
Muqian Chen, Xuebin Wang, Jinqiao Shi, Yue Gao, Can Zhao, \\ and Wei Sun
}

\section{Correction to: \\ Chapter "Towards Comprehensive Security Analysis \\ of Hidden Services Using Binding Guard Relays" \\ in: J. Zhou et al. (Eds.): Information and Communications \\ Security, LNCS 11999, \\ https://doi.org/10.1007/978-3-030-41579-2_30}

In the version of this paper that was originally published, the affiliation of Muqian Chen, Xuebin Wang, Yue Gao, and Can Zhao has been changed to: 'School of Cyber Security, University of Chinese Academy of Sciences, Beijing, China'. 